\section{Defining postoperative stability in children with radial polydactyly}

\author{
T. O. Engelhardt' ${ }^{1}$, G. Djedovic ${ }^{2}$, F. Pedross ${ }^{3}$ and H. Piza-Katzer ${ }^{2}$
}

The Journal of Hand Surgery

(European Volume)

2016, Vol. 41E(3) 275-280

(c) The Author(s) 2015

Reprints and permissions:

sagepub.co.uk/journalsPermissions.nav DOI: $10.1177 / 1753193415613583$

jhs.sagepub.com

\begin{abstract}
There is little fundamental data on paediatric metacarpophalangeal joint instability in radial polydactyly following surgical reconstruction. We evaluated 27 thumbs in a healthy paediatric population (Group A: eight girls and 19 boys with a mean age of 9.7 years (range 2.7-14.2)) and 12 thumbs following Wassel-IV reconstruction (Group B: eight girls and four boys with a mean age at follow-up of 10.6 years (range 2.713.2)). Metacarpophalangeal joint radial deviation, ulnar deviation on stress testing, interphalangeal joint and metacarpophalangeal joint alignment on posterior-anterior radiographs were measured and scored according to parameters defining joint instability. The aim of our study was to provide fundamental data on thumb metacarpophalangeal joint mobility patterns and alignment for further postoperative evaluations in children. The average ulnar deviation and radial deviation on stress testing of the healthy (Group A) metacarpophalangeal joints was $25^{\circ}\left(10^{\circ}-45^{\circ}\right)$ and $30^{\circ}\left(10^{\circ}-55^{\circ}\right)$, respectively. In the operated (Group B) thumbs, the ulnar deviation and radial deviation was greater at $35^{\circ}\left(10^{\circ}-55^{\circ}\right)$ and $30^{\circ}\left(10^{\circ}-70^{\circ}\right)$. Ulnar deviation (UD) of the proximal phalanx at the metacarpophalangeal joint on posterior-anterior radiographs was a mean of $10^{\circ}$ (range $-10^{\circ}-30^{\circ}$ ) in Group B; this was significantly greater than in Group $A$ at a mean of $5^{\circ}$ (range $\left.-5-20^{\circ}\right)(p=0.029)$. The mean radial alignment of the interphalangeal joint (distal phalanx relative to the proximal phalanx) was significantly higher in Group B $\left(15^{\circ}\right)$ than Group $A\left(0^{\circ}\right)(p=0.221)$. In the literature on radial polydactyly, cut off values defining metacarpophalangeal joint instability in children range from $5^{\circ}$ to $20^{\circ}$. According to our results, high but physiological metacarpophalangeal joint mobility of the thumb needs to be taken into consideration when evaluating children following reconstruction. Ulnar or radial deviation greater than $30^{\circ}$, in combination with the lack of a definite end point on metacarpophalangeal joint stress testing, may be regarded as unstable. Based on our study on healthy paediatric and reconstructed thumbs, comparison of joint stability with the healthy contralateral hand is recommended in order to define pathological instability.
\end{abstract}

Level of Evidence: II.

\title{
Keywords
}

Joint stability, radial polydactyly, Wassel classification, thumb

Date received: 2nd September 2014; revised: 13th September 2015; accepted: 26th September 2015

\section{Introduction}

Alignment and stability of the carpometacarpal, metacarpophalangeal (MP) and interphalangeal (IP) joints are regarded as major aims in the correction of congenital malformations of the thumb (BuckGramcko, 1998; Tada et al., 1983), such as Wassel Type IV radial polydactyly (RP) (Tada et al., 1983). Clinical studies have demonstrated that malalignment and instability have an important influence on the postoperative clinical result (Engelhardt et al., 2013; Naasan and Page 1994; Tada et al., 1983). In the surgical correction of RP, growing attention has been

\footnotetext{
${ }^{1}$ Handsurgery, Plastic and Aesthetic Surgery, Munich University Hospital, München, Germany

2Department of Plastic, Reconstructive and Aesthetic Surgery, Innsbruck Medical University

${ }^{3}$ Department of Medical Statistics, Innsbruck Medical University, Innsbruck, Austrialnnsbruck, Austria
}

\section{Corresponding author:}

T. O. Engelhardt, Handsurgery, Plastic and Aesthetic Surgery, Munich University Hospital, Ludwig-Maximilians University,

Marchioninistraße 15, 81377 München, Germany.

Email: timm.engelhardtamed.uni-muenchen.de 
directed to avoiding malalignment and instability of the MP joint of the operated thumb to improve clinical outcomes (Patel et al., 2013; Tada et al., 1983) (Figure 1).

Chronic MP joint instability following reconstruction of a Wassel type IV RP may impair prehensile function and contribute to late onset osteoarthritis. Revision surgery and MP joint stabilization procedures may be needed. Unfortunately our understanding and diagnosis of thumb MP joint instability in these cases is limited.

Scoring systems have included MP joint stability as a major parameter. The most frequently applied clinical scores are: the Tada Score (Tada et al., 1983); the modified Tada score (Ogino et al., 1996); the Score of the Japanese Society for Surgery of the Hand (Horii et al., 2009); and the Hung score (Hung et al., 1996).

In the literature on congenital malformations of the thumb, criteria defining pathologic MP joint instability have been inconsistent, ranging from $>5^{\circ}$ to $>40^{\circ}$ (Horii et al., 2009; Hung et al., 1996; Maillet et al., 2007; Ogino et al., 1996; Tada et al., 1983; Yen et al., 2006) of radial or UD on manual stress testing. In adults, passive deviation of the MP joint greater than $20^{\circ}-30^{\circ}$, in combination with lack of a definite end point on stress examination, is considered pathological (Malik et al., 2009; Smith and Lister, 2002). In children, following reconstruction of RP relevant data on radial and UD of the thumb MP joint is limited.

The purpose of our study was to assess MP joint stability of the thumb, and MP and IP joint motion patterns in normal children and in patients following reconstruction for Wassel IV RP.

\section{Materials and methods}

We assessed the thumbs in 27 normal children (Group A) and in 12 patients following reconstruction of Wassel IV RP (Group B). The inclusion criteria for Groups A and B were: normal thumbs (Group A); and thumbs following reconstruction of Wassel IV thumbs (Group B). The exclusion criteria for both groups were: any history of upper extremity trauma of the extremity examined; revision surgery of the thumb (Group B); and other musculoskeletal diseases and congenital malformations of the upper extremity (Group A).

In Group A $(N=27)$ there were 19 boys and eight girls with a mean age of 9.7years (range 2.7 to 14.2). In Group $B(N=12)$ there were four boys and eight girls. They were operated upon at a mean age of 1.7 years (range 0.6 to 8.6 ). They were followed up at a mean of 8.9 years (range 1.4 to 12) post-operatively. Thus, at assessment, the children in Group B had a mean age of 10.6 years (range 2.7 to 13.2) (Table 1).

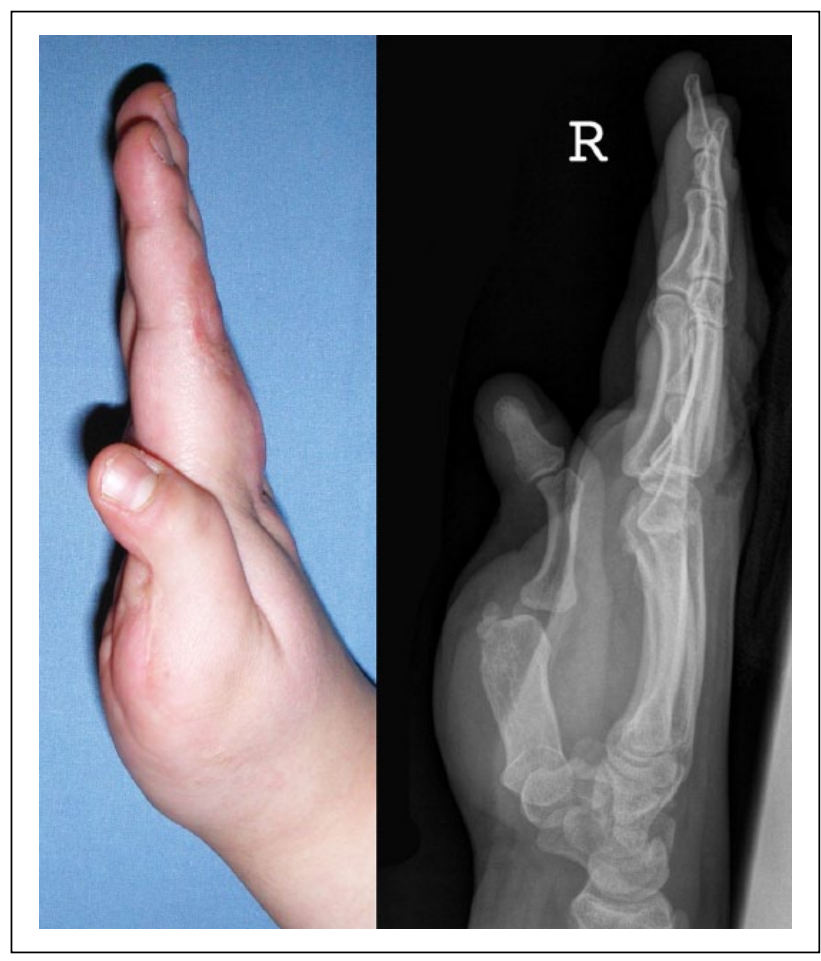

Figure 1. Poor clinical result (left) following resection of a radial hypoplastic accessory thumb in RP Wassel type IV in another institution without articular reconstruction in a 23-year-old female (22 years postoperative). Postoperative standard radiograph (right) showing malalignment of the MP joint (ulnar alignment) and IP joint (radial alignment) including ulnar IP joint and radial MP joint instability.

Table 1. Patient demographic data.

\begin{tabular}{lll}
\hline Study group & Group A & Group B \\
\hline Thumbs & Normal & Wasse IV \\
Male & 19 & 4 \\
Female & 8 & 8 \\
Average age (years) & $9.7(2.7-14.2)$ & $10.6(2.7-13.2)$ \\
Follow-up (years) & no surgery & $6.7(1.4-12)$ \\
\hline
\end{tabular}

\section{Surgical treatment}

The patients in Group B had reconstruction with resection of the hypoplastic radial thumb and anatomical stabilization of the MP joint, as described by Engelhardt et al. (2013). There were no cases with a pre-operative zigzag deformity of Wassel IV thumbs Iradial deviation at the level of the IP and UD at the level of the MP joint of the remaining thumb, also known as the divergent/convergent thumb).

\section{Assessments}

Radial/ulnar alignment of the thumb MP joints at rest was examined radiologically with a postero-anterior 


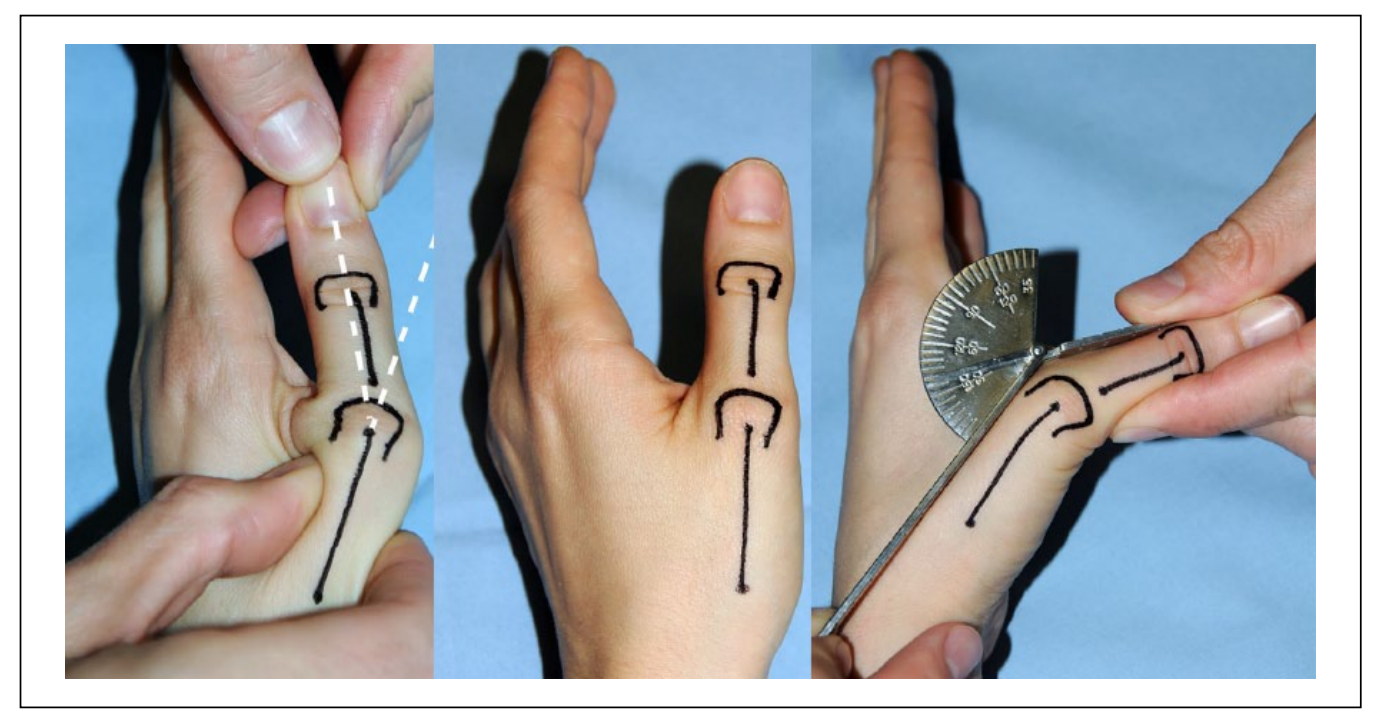

Figure 2. Ulnar (right) and radial (left) manual stress testing: clinical evaluation of MP joint stability of left healthy thumb in a 14-year-old female. Neutral MP joint without stress testing (middle).

radiograph and measured with a standard goniometer. We recorded the results as means of two measurements performed by two different observers of the treating team. Since the IP joint may show additional deviation following Wassel IV reconstruction, the IP joints were also examined accordingly.

Passive RD and UD of the MP joint were tested manually on stress examination of the MP joint in $20^{\circ}$ of flexion until an end point was reached, as previously described (Malik et al., 2009) using a standard goniometer. The degree of deviation was measured manually in $5^{\circ}$ intervals by the examiners. Moderate pressure was applied to the lateral aspect of the proximal phalanx, while the thumb metacarpal was held in neutral. Stress examination pressure of $2 \mathrm{~kg}$ was applied and controlled by using a conventional pinch meter. The presence (+) or lack (-) of a definite end point on ulnar or radial stress testing was evaluated. We recorded the results as means of two measurements performed by two different observers (Figure 2).

The thumb MP joints were rated as stable or unstable according to published criteria by Tada et al., Ogino et al. and Japanese Society for Surgery of the Hand by Horii et al. (Horii et al., 2009; Hung et al., 1996; Ogino et al., 1996; Tada et al., 1983). These scores range from $>5^{\circ}$ to $>40^{\circ}$ of increased deviation of the MP joint.

\section{Statistical analysis}

The non-parametric Mann-Whitney $U$ Test and Wilcoxon signed rank tests were applied to evaluate clinical parameters between Groups A and B, including
Spearman-Rho for the evaluation of non-parametric correlations between and within both groups.

\section{Results}

\section{Alignment and stability}

The measurements for MP and IP joint alignment, as well as MP joint stability, are shown in Table 2.

The mean radial alignment of the IP joint was higher in Group B $\left(15^{\circ}\right)(p=0.221)$ compared with Group $A\left(0^{\circ}\right)$. The mean ulnar alignment at the level of the MP joint in Group B $\left(10^{\circ}\right)$ was two times higher than in Group A $\left(5^{\circ}\right)(p=0.029)$. The intra-observer variability showed an intraclass correlation coefficient (ICC) of 0.7797 , the inter-observer variability revealed an ICC of 0.7532 .

When assessing joint stability, the mean MP joint UD/RD in healthy thumbs was $25^{\circ} / 30^{\circ}$. UD was significantly higher in Group $B\left(35^{\circ}\right)$ following Wassel IV reconstruction compared with Group A $\left(25^{\circ}\right)$ $(p=0.011)$. The intra-observer variability showed an ICC of 0.7083 ; the inter-observer variability revealed an ICC of 0.7296 . In all patients (Groups A and B) there was a definite end point on stress testing the MP joint, indicating intact collateral ligaments (Malik et al., 2009).

In healthy thumbs, there was a significant positive correlation between MP joint UD and RD, that is, an increased UD correlated with increased RD $(p=0.013, r$ : 0.48). In Group B there was a significant correlation between MP joint UD and IP joint radial mal-alignment.

Applying the criteria of Tada et al. (1983), Ogino et al. (1996), Hung et al. (1996) and Japanese Society for 
Table 2. Clinical results.

\begin{tabular}{|c|c|c|c|}
\hline & $\begin{array}{l}\text { Group A } \\
\text { Healthy thumbs }\end{array}$ & $\begin{array}{l}\text { Group B } \\
\text { Wassel IV }\end{array}$ & $\begin{array}{l}\mathrm{B} / \mathrm{A} \\
\text { Difference }\end{array}$ \\
\hline & $N=27$ & $N=12$ & \\
\hline MP joint radial deviation & $30^{\circ}(10-55)^{1}$ & $30^{\circ}(10-70)$ & $p 0.822$ \\
\hline MP joint ulnar deviation & $25^{\circ}(10-45)$ & $35^{\circ}(10-55)^{2}$ & $p 0.011^{3}$ \\
\hline MP joint end point on stress test & $N=27$ & $N=12$ & \\
\hline MP joint ulnar alignment & $5^{\circ}(-5-20)$ & $10^{\circ}(-12-30)$ & $p 0.0293$ \\
\hline IPJ radial alignment & $0^{\circ}(-15-5)$ & $15^{\circ}(5-0)$ & $p 0.221$ \\
\hline
\end{tabular}

1Significant correlation to ulnar deviation in Group A: $p=0.013, \mathrm{r}: 0.480$.

2Significant correlation to radial alignment at IP-joint level in Group B: $p=0.001, r: 0.823$.

${ }^{3}$ Significant for $p<0.05$.

IPJ: interphalangeal joint; MP: metacarpophalangeal.

Surgery of the Hand /Horii (Horii et al., 2009) for instability, 12 (Tada), ten (Ogino), ten (Hung) and six (Japanese Society for Surgery of the Hand) thumb MP joints were rated as 'unstable' in Group B (Table 3). When these scores were applied in healthy thumbs (Group A) all 27 were classified as 'unstable' according to Tada's criteria, and 20 according to the criteria of both Ogino et al. (1996) and Hung et al. (1996). Applying the Japanese Society for Surgery of the Hand score (Horii et al., 2009) three thumbs were classified as unstable.

\section{Discussion}

Alignment, size and stability of the thumb are considered essential parameters for the postoperative early development and maintenance of prehensile function (Buck-Gramcko, 1998; Tada et al., 1983). Instability of the MP joint following reconstruction in children with congenital malformations of the hand correlates with thumb deformity (Buck-Gramcko, 1998; Naasan and Page, 1994; Tada et al., 1983), which alters prehensile function.

Although various articles on clinical and radiological results report on MP joint stability in children, there is great diversity regarding its definition. Published results focusing on the treatment of RP are commonly presented as score results rather than in degrees of $\mathrm{RD}$ or UD. In the treatment of RP, the most common parameters of MP joint stability are: TADA Score (Tada et al., 1983) - instability: RD $>5^{\circ}$; modified Tada Score by Ogino (Ogino et al., 1996) - instability: RD $>20^{\circ}$; Score proposed by Hung (Hung et al., 1996) - instability: RD $>20^{\circ}$; Score of the Japanese Society for Surgery of the Hand (Horii et al., 2009) - instability $>40^{\circ}$.

In adult populations, passive MP joint deviation greater than $20^{\circ}-30^{\circ}$ has been considered pathological (Malik et al., 2009; Smith and Lister, 2002). Another method to detect instability is the radiological evaluation of phalangeal translation of the proximal phalanx on stress testing (McKeon et al., 2013) (Figure 3).

There is a lack of fundamental data to differentiate between stability, physiologic laxity and instability of malformed, operated as well as healthy paediatric MP joints. In a larger series (Hung et al., 1996; Larsen and Nicolai, 2005; Maillet et al., 2007; Naasan and Page, 1994; Ogino et al., 1996; Tada et al., 1983; Yen et al., 2006) only Hung et al. (Hung et al., 1996) provides data regarding UD of the paediatric MP joint in numbers (degrees) rather than score results.

In our study, in normal children there was a mean MP joint RD of $30^{\circ}$ and UD of $25^{\circ}$ with definite end points (Malik et al., 2009). When traditional RP scores such as the Tada Score were applied (Tada et al., 1983), all of the healthy MP joints were classified as unstable. These results may be misleading.

Greater physiological joint mobility in the hand has been reported in children, depending on age and race (Azevedo and Santos, 1982; de Smet et al., 1993; Kuo et al., 2009). Significant correlation between gender and motion patterns and morphology of the thumb have been reported (Yoshida et al., 2003). This further confuses assessment of MP joint instability (de Kraker et al., 2009).

According to our results, physiologic normal joint deviation of the MP joint seems higher than has previously been described as instability in published scores. A simple cut-off value of $20^{\circ}$ to define radial or ulnar instability may thus not be adequate in the evaluation of surgical results in children.

A large age range in our paediatric population may be regarded as weakness of our study. It cannot be denied that precise clinical assessment of instability in small children is difficult and technically demanding. Therefore, clinical results following stress test examination were measured in $5^{\circ}$ intervals.

Our findings indicate that any passive RD or UD up to $30^{\circ}$ should be regarded as physiological in children 
Table 3. Radial MP joint instability according to published criteria.

\begin{tabular}{lll}
\hline Radial instability & $\mathrm{A}$ & $\mathrm{B}$ \\
\cline { 3 - 3 } & Normal thumbs & Wassel IV \\
\hline scores applied & $N=27$ & $N=12$ \\
Tada: MP joint $>5^{\circ}$ (Tada et al., 1983) & Instability $N=27$ & Instability $N=12$ \\
Ogino: MP joint $>20^{\circ}$ (Ogino et al., 1996) & Instability $N=20$ & Instability $N=10$ \\
Hung: MP joint $>20^{\circ}$ (Hung et al., 1996) & Instability $N=20$ & Instability $N=10$ \\
JSSH: MP joint $>41^{\circ}$ (Horii et al., 2009) & Instability $N=3$ & Instability $N=6$ \\
\hline
\end{tabular}

JSSH: Japanese Society for Surgery of the Hand; MP: metacarpophalangeal.

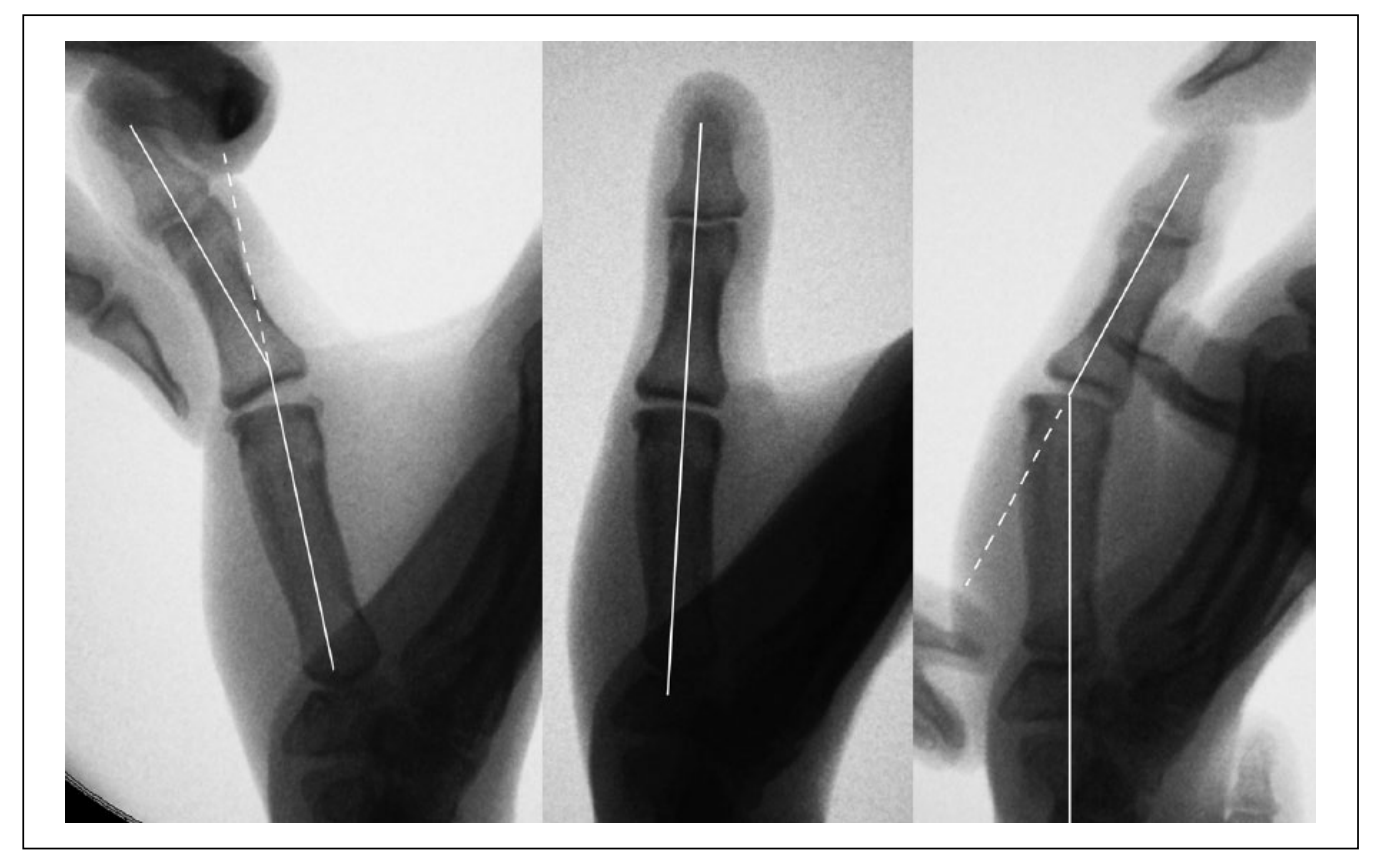

Figure 3. Posterior-anterior radiograph of a healthy right thumb in another 16-year-old healthy male (not included in Group A) with neutral alignment at rest (middle) and physiological $27^{\circ}$ of passive MP joint, UD under manual MP joint radial stress testing (right).

up to 14 years old. Due to our clinical study, we recommend that any possible instability of the MP joint in children requires careful assessment against the opposite thumb.

\section{Declaration of conflicting interests}

The authors declared no potential conflicts of interest with respect to the research, authorship, and/or publication of this article.

\section{Funding}

The authors received no financial support for the research, authorship, and/or publication of this article.

\section{References}

Azevedo ES, Santos MC. Joint mobility in children: a population study. Acta Anthropogenet. 1982, 6: 33-43.
Buck-Gramcko D. Congenital malformations of the hand forearm. London, Churchill Livingstone, 1998.

de Kraker M, Selles RW, Molenaar TM et al. Palmar abduction measurements: reliability and introduction of normative data in healthy children. J Hand Surg Am. 2009, 34: 1704-8.

de Smet L, Urlus M, Spriet A et al. Metacarpophalangeal and interphalangeal flexion of the thumb: influence of sex and age, relation to ligamentous injury. Acta Orthop Belg. 1993, 59: 357-9.

Engelhardt TO, Baur EM, Pedross F et al. Supporting the collateral ligament complex in radial polydactyly type Wassel IV. J Plast Reconstr Aesthet Surg. 2013, 66: 104-12.

Horii E, Hattori T, Koh S et al. Reconstruction for Wassel type III radial polydactyly with two digits equal in size. J Hand Surg Am. 2009, 34: 1802-7.

Hung L, Cheng JC, Bundoc R et al. Thumb duplication at the metacarpophalangeal joint. Management and a new classification. Clin Orthop Relat Res. 1996, 323: 31-41.

Kuo L-C, Cooney WP, An K-N et al. Effects of age and gender on the movement workspace of the trapeziometacarpal joint. Proc IMechE, Part H. 2009, 223: 133-42. 
Larsen M, Nicolai JPA. Long-term follow-up of surgical treatment for thumb duplication. J Hand Surg Br. 2005, 30: 276-81.

Maillet M, Fron D, Martinot-Duquennoy V et al. Results after surgical treatment of thumb duplication: a retrospective review of 33 thumbs. J Child Orthop. 2007, 1: 135-41.

Malik AK, Morris T, Chou D et al. Clinical testing of ulnar collateral ligament injuries of the thumb. J Hand Surg Eur. 2009, 34: 363-6.

McKeon KE, Gelberman RH, Calfee RP. Ulnar collateral ligament injuries of the thumb: phalangeal translation during valgus stress in human cadavera. J Bone Joint Surg Am. 2013, 95: 881-7.

Naasan A, Page RE. Duplication of the thumb. A 20 -year retrospective review. J Hand Surg Br. 1994, 19: 355-60.

Ogino T, Ishii S, Takahata $\mathrm{S}$ et al. Long-term results of surgical treatment of thumb polydactyly. J Hand Surg Am. 1996, 21: 478-86.
Patel AUC, Tonkin MA, Smith BJ et al. Factors affecting surgical results of Wassel type IV thumb duplications. J Hand Surg Eur. 2014, 39: 934-43.

Smith PJ, Lister G. Lister's The hand. 4th Edn. London, Churchill Livingstone, 2002.

Tada K, Yonenobu K, Tsuyuguchi Y et al. Duplication of the thumb. A retrospective review of two hundred and thirty-seven cases. J Bone Joint Surg Am. 1983, 65: 584-98.

Yen $\mathrm{CH}, \mathrm{Chan} \mathrm{WL}$, Leung HB et al. Thumb polydactyly: clinical outcome after reconstruction. J Orthop Surg (Hong Kong). 2006, 14: 295-302.

Yoshida R, House HO, Patterson RM et al. Motion and morphology of the thumb metacarpophalangeal joint. J Hand Surg Am. 2003, 28: 753-7. 